\title{
A Preliminary Argument for the Selective Use of the Robiscek Weave
}

\author{
John Ikonomidis ${ }^{1}$ \\ ${ }^{1}$ University of North Carolina
}

June 10, 2021

\section{A Preliminary Argument for the Selective Use of the Robicsek Weave}

John S. Ikonomidis MD, PhD

Division of Cardiothoracic Surgery, University of North Carolina at Chapel Hill

Word Count: 886

References: 4

Address correspondence to:

John S. Ikonomidis MD, PhD

Professor and Chief,

Division of Cardiothoracic Surgery

University of North Carolina at Chapel Hill

3034 Burnett Womack Building

160 Dental Circle,

Chapel Hill, NC

27599

e-mail: john_ikonomidis@med.unc.edu

Tel: (919) 966-3381

Proper execution of median sternotomy and its subsequent closure are critical to the success of cardiac surgical outcomes. It is essential that the sternum be divided directly in the midline, and table fractures must be avoided if at all possible by avoiding excessive spreading if the sternum for exposure of the heart. Multiple methods have been described regarding primary sternal closure technique, but the conventional technique of wire circlage, either linear or figure-of-eight, has endured and is also the most cost-effective. Sternal wound complications have an incidence of $0.8 \%$ to $1.5 \%$ patients, and this number rises to as high as $8 \%$ when bilateral internal mammary artery harvest is undertaken. Further established risk factors for deep sternal wound complications include breaches in sterility in the operating room, lengthy operations, reexploration for bleeding, undrained retrosternal hematoma, incomplete wound closure, obesity, advanced age, diabetes, chronic obstructive pulmonary disease, hospital acquired pneumonias, renal failure, requirement for dialysis, and prolonged mechanical ventilation. Mortality from sternal dehiscence and related complications ranges from $6 \%$ to $70 \%$. It is generally felt that early treatment reduces mortality. ${ }^{1}$ 
Deep sternal wound complications and dehiscence were once thought to be highly feared and challenging complications of cardiac surgery. Modern primary closure techniques, tissue flap coverage options, and negative pressure wound therapy have made these complications more manageable. Nevertheless, it behooves surgeons to avoid this complication due to its considerable negative clinical impact.

There are many methods currently available for reconstruction of the sternum after its dehiscence, the most common of which is the sternal weave first described by Robicsek and colleagues in $1977 .{ }^{2}$ This technique is often used to reinforce the sternum with primary sternal closure in instances where the sternotomy was off the midline leaving a thin weak section of sternum on one side or where some fracturing has occurred, but has also been used as a first line for sternal reconstruction after its dehiscence from primary closure. Data are not available regarding the overall success rate of reinforcement using the Robicsek weave, but at least one multicenter, randomized controlled trial showed that in patients with an increased risk for sternal instability and wound infection after cardiac surgery, sternal reinforcement using the Robicsek technique prior to primary sternal closure did not reduce dehiscence rate. ${ }^{3}$

In addition to the above, antecedent sternal weaving weave may complicate further attempts at sternal closure should dehiscence recur. In this issue of the Journal of Cardiac Surgery, ${ }^{4}$ Seyrek et al. conducted a retrospective review of patients at a single institution with noninfectious sternal dehiscence (NISD) after median sternotomy who received thermoreactive nitinol clips (TRNC) for sternal closure. The authors studied 34 cases who received TRNC treatment between December 2009 and January 2020 out of 283 patients with NISD who underwent sternal refixation. These cases were divided into two groups: patients who had a previously failed Robicsek procedure before TRNC treatment (group A, n=11) and patients who had been directly referred to TRCN treatment (group $\mathrm{B}, \mathrm{n}=23$ ). The results showed that the postoperative complication rate and length of hospital stay was significantly higher with use of the Robicsek weave. Further, operative time was significantly shorter and blood loss was significantly lower in patients referred for sternal refixation without having first undergone a Robicsek weave.

Part of the reason for the above results may lie with the surgical requirements for performance of the Robicsek weave. Substernal and lateral dissection is required to define the margins of the sternum before placing the weave. This increases the technical difficulty of the reclosure operation and puts the patient at risk for inadvertent injury to the heart, great vessels, and other mediastinal structures. This dissection may also compromise blood flow to the sternal half. Further, intercostal arteries may be squeezed by weave as it runs anteriorly and posteriorly around the ribs, which may occlude blood supply to the sternum. This could worsen pre-existing ischemia, which would delay sternal healing, promote bacterial colonization, and cause bone necrosis and additional sternal fragmentation, thus complicating any additional closure attempts.

Use of TRNC may represent an advance in sternal reconstruction therapy due to the simplicity of use and lack of requirement for a complex mediastinal dissection prior to application. The authors contend that a previously failed Robicsek procedure caused significantly higher morbidity, additional operative risk and lower success rate in later TRNC treatment of high-risk cases and hence speculate that patients at high risk for sternal separation should proceed directly to TRNC treatment. In the light of the above study, this approach seems reasonable, but a prospective trial should be considered to provide the definitive answer.

\section{References}

1. Kaul P. Sternal reconstruction after post-sternotomy mediastinitis. J Cardiothorac Surg 2017; 12:94-104.

2. Robicsek F, Daugherty HK, Cook JW. The prevention and treatment of sternum separation following open-heart surgery. J Thorac Cardiovasc Surg 1977;73:267-268.

3. Schimmer C, Reents W, Berneder S, Eigel P, Sezer O, Scheld H, Sahraoui K, Gansera B, Deppert O, Rubio A, Feyrer R, Sauer C, Elert O, Leyh R. Prevention of sternal dehiscence and infection in high-risk patients: a prospective randomized multicenter trial. Ann Thorac Surg 2008;86:1897-1904.

4. Seyrek Y, Akkuş M. What is the impact of a previously failed Robicsek repair in the subsequent treatment of sternal dehiscence with thermoreactive nitinol clips? Which sternal refixation method should be preferred 
in high-risk cases? J Card Surg, in press. 